\title{
23
}

\section{Interactive Structural Optimization with Quasi-Newton Algorithms}

\author{
C. Pedersen \& P. Thoft-Christensen
}

Aalborg University, Sohngaardsholmsvej 57, DK-9000 Aalborg, Denmark

\begin{abstract}
This paper presents a prototype of an interactive optimization system IROS (Interactive Reliability-Based Optimization System), where the designer during the optimization process is able to adjust simple bounds, fix or relax design variables and include/exclude constraints. The optimization is based on a modified quasi-Newton algorithm, which includes a subproblem that minimizes the condition number of the approximate Hessian matrix in each iteration.
\end{abstract}

Keywords: Interactive Optimization, Quasi-Newton Algorithms, Reliability-Based Optimization.

\section{INTRODUCTION}

During the last decades, one of the most important renewals in the fields of structural and especially mechanical design has been the innovation of fully automatic systems and computer based analysis tools. However, fully automated systems are generally not considered feasible or preferable within the field of structural optimization. Therefore, interactive systems are assumed to be used within several engineering fields in the future.

Considering structural constrained optimization problems only, the class of algorithms based on quasi-Newton schemes and Lagrange multipliers have proven to be most efficient due to their relatively low number of function and gradient evaluations.

This paper presents a prototype of an interactive optimization system based on a quasiNewton algorithm, where the designer during the optimization process is able to adjust simple bounds, fix or relax design variables and include/exclude constraints. The optimization is based on a modified quasi-Newton algorithm, which includes a subproblem that minimizes the condition number of the approximate Hessian matrix in each iteration. The purpose is to minimize the condition number of the approximate Hessian matrix and thereby stabilize the determination of the search direction in each iteration after major interactions.

The interactive optimization is carried out through a shell called IROS (Interactive Reliability-Based Optimization System). IROS is mainly intended to constitute a platform for testing of various algorithms and interactive capabilities rather than being a commercial programme. In order to demonstrate some of the facilities of an interactive optimization system, a simple example of a reliability-based optimization of a plane portal frame of steel with I-shaped cross-sections is shown. 


\section{RELIABILITY-BASED OPTIMIZATION PROBLEM}

In order to introduce the terminology used, the general formulation of a non-linear constrained optimization problem is stated as

$$
\begin{array}{cll}
\min _{\mathbf{z}} & C(\mathbf{z}) & \\
\text { s.t. } & c_{j}(\mathbf{z})=0 & j=1, \ldots, m_{e} \\
& \hat{c}_{j}(\mathbf{z}) \geq 0 & j=1, \ldots, m_{i} \\
& z_{i}^{\min } \leq z_{i} \leq z_{i}^{\max } & i=1, \ldots, n
\end{array}
$$

where $\mathbf{z}^{T}=\left(z_{1}, z_{2}, \ldots, z_{n}\right)$ denote the design variables. Introducing the Lagrange multipliers $\lambda_{i}$, the Lagrange function $L(\cdot)$ is defined as (5), where the $2 n$ simple bounds are included as inequality constraints of the standard form (3).

$L(\mathbf{z}, \boldsymbol{\lambda})=C(\mathbf{z})-\sum_{j=1}^{m_{e}} \lambda_{j} c_{j}(\mathbf{z})-\sum_{j=1}^{m_{i}+2 n} \lambda_{m_{e}+j} \hat{c}_{j}(\mathbf{z})$

The reliability-based optimization problem on element level is obtained when a subset of the constraints (3) is of the form

$\hat{c}_{j}(\mathbf{z})=\beta_{j}(\mathbf{z})-\beta_{j}^{\min }$

Introducing the stochastic variables $\mathbf{X}$, the corresponding standardized independent and normally distributed $\mathbf{U}$-space obtained by the transformation $\mathbf{X}=\mathbf{T}(\mathbf{U})$ and the failure function $g_{j}(\mathbf{x}, \mathbf{z})$, the probability of failure $P_{f}=\Phi^{-1}\left(\beta_{j}\right)$ can be approximated by the FORM solution, where the reliability index $\beta_{j}(\cdot)$ in $(6)$ is obtained from

$$
\begin{aligned}
\beta_{j}=\min _{\mathbf{u}} & \sqrt{\mathbf{u}^{T} \mathbf{u}} \\
\text { s.t. } & g_{j}(\mathbf{T}(\mathbf{u}), \mathbf{z})=0
\end{aligned}
$$

A closer description can be found in Madsen et al. (1986) and others.

Using a quasi-Newton algorithm, optimum $\mathbf{z}^{*}$ is found iteratively as the limit of the sequence $\left\{\mathbf{z}^{(k)}, k=1,2, \ldots\right\}$. The iterate $\mathbf{z}^{(k+1)}$ is obtained as $\mathbf{z}^{(k+1)}=\mathbf{z}^{(k)}+\alpha \mathbf{d}$, where $\mathbf{d}$ denotes the search direction while the step length $\alpha$ is obtained from a one-dimensional line search in the direction $\mathbf{d}$ in a merit function $\phi(\cdot)$.

The search direction $\mathbf{d}$ in each iteration is determined as the solution to a sequential quadratic programming (SQP) subproblem of the form

$$
\begin{array}{lll}
\min _{\mathbf{d}} & \nabla_{z} C\left(\mathbf{z}^{(k)}\right)^{T} \mathbf{d}+\frac{1}{2} \mathbf{d}^{T} \mathbf{B}^{(k)} \mathbf{d} & \\
\text { s.t. } & c_{j}\left(\mathbf{z}^{(k)}\right)+\nabla_{z} c_{j}\left(\mathbf{z}^{(k)}\right)^{T} \mathbf{d}=0 & j=1, \ldots, m_{e} \\
& \hat{c}_{j}\left(\mathbf{z}^{(k)}\right)+\nabla_{z} \hat{c}_{j}\left(\mathbf{z}^{(k)}\right)^{T} \mathbf{d} \geq 0 & j=1, \ldots, m_{a} \\
& z_{i}^{(k)}-z_{i}^{\min } \leq d_{i} \leq z_{i}^{\max }-z_{i}^{(k)} & i=1, \ldots, n
\end{array}
$$

where only a subset of all $m_{i}$ inequality constraints in (3) is included in the linearized constraints (11). $\mathbf{B}^{(k)}$ denotes a positive definite matrix which gradually is updated by 
use of a quasi-Newton scheme (refer to section 4) to approach the Hessian matrix of the Lagrange function $L(\cdot)$.

Introducing the column vector $\mathbf{h}(\mathbf{z})$ in which all equality and active inequality constraints are assembled, the solution of (9)-(12) is obtained from the linear system

$$
\left[\begin{array}{cc}
\mathbf{B}^{(k)} & -\boldsymbol{\nabla}_{z} \mathbf{h}\left(\mathbf{z}^{(k)}\right) \\
-\boldsymbol{\nabla}_{z} \mathbf{h}\left(\mathbf{z}^{(k)}\right)^{T} & \mathbf{0}
\end{array}\right]\left[\begin{array}{c}
\mathbf{d} \\
\boldsymbol{\lambda}^{(k+1)}
\end{array}\right]=\left[\begin{array}{c}
-\boldsymbol{\nabla}_{z} f\left(\mathbf{z}^{(k)}\right) \\
\mathbf{h}\left(\mathbf{z}^{(k)}\right)
\end{array}\right]
$$

Standard solution techniques are outlined Ringertz (1993) or Golub \& Van Loan (1989), while a modification ensuring that the linearized constraints (10)-(11) on $\mathbf{d}$ are consistent can be found in Powell (1977).

\section{INTERACTIVE PROCEDURES}

In order to provide the interactive facilities of include/exclude constraints, change simple bounds and the change current value and fix/relax design variables, modifications of standard quasi-Newton algorithms are necessary. A qualitative discussion of various possibilities can be found in Pedersen \& Thoft-Christensen (1993).

Considering active set changes with respect to the design variables (i.e. only a subset of all $n$ design variables $\mathbf{z}$ is allowed to vary), a straightforward strategy is to adjust the dimension of the subproblem (9)-(12). Unfortunately, a frequent change of the dimension of the Hessian matrix $\mathbf{B}^{(k)}$ results in loss of already obtained information in cases where temporarily fixed design variables are re-included in the optimization problem. Furthermore, the choice of the additional rows and columns must fulfil certain requirements in order to preserve positive definiteness of $\mathbf{B}^{(k)}$.

An alternative approach is to include an additional equality constraint per fixed design variable in (9)-(12) whereby the full dimension of $\mathbf{B}^{(k)}$ and positive definiteness is preserved. Thus, from the modified solution scheme written below for the search direction subproblem in the case where design variables $z_{i}$ is fixed, it is easily seen that $d_{i}$ is equal to zero.

$$
\left[\begin{array}{ccc}
\mathbf{B}^{(k)} & -\boldsymbol{\nabla}_{z} \mathbf{h}\left(\mathbf{z}^{(k)}\right) & \mathbf{e}_{i} \\
-\boldsymbol{\nabla}_{z} \mathbf{h}\left(\mathbf{z}^{(k)}\right)^{T} & \mathbf{0} & \mathbf{0} \\
\mathbf{e}_{i}^{T} & \mathbf{0} & 0
\end{array}\right]\left[\begin{array}{c}
\mathbf{d} \\
\boldsymbol{\lambda}^{(k+1)}
\end{array}\right]=\left[\begin{array}{c}
-\nabla_{z} f\left(\mathbf{z}^{(k)}\right) \\
\mathbf{h}\left(\mathbf{z}^{(k)}\right) \\
0
\end{array}\right]
$$

where the column vector $\mathbf{e}_{i}=\{0, \ldots 0,1,0, \ldots 0\}^{T}$ contains zeroes except at the $i$ th position. Due to the presence of the additional constraint, the corresponding active constraint set $\mathbf{h}\left(\mathbf{z}^{(k)}\right)$ is different from the active set that corresponds to the standard problem where all design variables are allowed to vary. Finally, the last element in the vector $\boldsymbol{\lambda}^{(k+1)}$ is seen to correspond to the artificial constraint $d_{i}=0$.

With respect to the interactive inclusion/exclusion of constraints, change of current design point and simple bounds, the major difficulties originates from the fact that the Hessian matrix $\mathbf{B}^{(k)}$ and the gradients may be inconsistent. Since the search direction $\mathbf{d}$ obtained from the subproblem (9)-(12) with an ill-conditioned Hessian matrix (ill-conditioned in terms of a large condition number - i.e. the ratio between the largest and smallest eigenvalues) is strongly dependent upon the actual values of the gradients, a minimization of $\operatorname{cond}\left(\mathbf{B}^{(k+1)}\right)$ is considered in the next section. 


\section{REVISION OF THE APPROXIMATE HESSIAN MATRIX}

The revision of the approximate Hessian matrix $\mathbf{B}^{(k)}$ is required to satisfy the quasi-Newton condition - see e.g. Gill et al. (1981)

$\mathbf{B}^{(k+1)} \mathbf{p}^{(k)}=\mathbf{q}^{(k)}$

where $\mathbf{p}^{(k)}$ is the difference between the current and previous design point

$\mathbf{p}^{(k)}=\mathbf{z}^{(k)}-\mathbf{z}^{(k-1)}=\alpha \mathbf{d}^{(k)}$

while $\mathbf{q}^{(k)}$ is dependent upon the difference between the current and previous derivatives of the Lagrangian $L(\mathbf{z}, \boldsymbol{\lambda})$. Among the most frequently used definitions of $\mathbf{q}^{(k)}$ is

$\mathbf{q}^{(k)}=\theta_{k}\left(\boldsymbol{\nabla}_{z} L\left(\mathbf{z}^{(k)}, \boldsymbol{\lambda}^{(k)}\right)-\boldsymbol{\nabla}_{z} L\left(\mathbf{z}^{(k)}, \boldsymbol{\lambda}^{(k)}\right)\right)+\left(1-\theta_{k}\right) \mathbf{B}^{(k)} \mathbf{p}^{(k)}$

proposed by Powell (1977) where only the latest set of Lagrange multipliers is used and the additional parameter $\theta_{k}$ is introduced in order to preserve positive definiteness. In Powell (1977), $\theta_{k} \in[0,1]$ is chosen in such manner that $\mathbf{q}^{(k)^{T}} \mathbf{p}^{(k)} \geq 0.2 \mathbf{p}^{(k)^{T}} \mathbf{B}^{(k)} \mathbf{p}^{(k)}$ which ever since has been widely used in numerous algorithms - e.g. NLPQL by Schittkowski.

With the above definition of $\mathbf{p}^{(k)}$ and $\mathbf{q}^{(k)}, \mathbf{B}^{(k)}$ is usually revised using a symmetric rank-two update formula. In accordance with Gill et al. (1981) and Luenberger (1984) the one-parameter Broyden family update formula may be written as

$\mathbf{B}^{(k+1)}=\mathbf{B}^{(k)}-\frac{\mathbf{B}^{(k)} \mathbf{p}^{(k)} \mathbf{p}^{(k)} \mathbf{B}^{(k)}}{\mathbf{p}^{(k)^{T}} \mathbf{B}^{(k)} \mathbf{p}^{(k)}}+\frac{\mathbf{q}^{(k)} \mathbf{q}^{(k)^{T}}}{\mathbf{q}^{(k)^{T}} \mathbf{p}^{(k)}}+\phi_{k}\left(\mathbf{p}^{(k)^{T}} \mathbf{B}^{(k)} \mathbf{p}^{(k)}\right) \mathbf{w} \mathbf{w}^{T}$

where $\mathbf{w}$ is defined as

$\mathbf{w}=\frac{\mathbf{q}^{(k)}}{\mathbf{q}^{(k)^{T}} \mathbf{p}^{(k)}}-\frac{\mathbf{B}^{(k)} \mathbf{p}^{(k)}}{\mathbf{p}^{(k)^{T}} \mathbf{B}^{(k)} \mathbf{p}^{(k)}}$

Choosing $\phi_{k}=1$ the formula (18) is termed the DFP update while $\phi_{k}=0$ is resulting in the efficient and even more commonly used BFGS update formula. Furthermore, since both the DFP and BFGS updates satisfy (15), it is easily seen by premultiplying (19) by $\mathbf{p}^{(k)}$ that any choice of $\phi_{k}$ also satisfies (15).

In an interactive environment constraints are included/excluded, the current design point changed manually, etc. Therefore, a well-conditioned Hessian matrix is desirable in order to obtain a stable solution in terms of a feasible descent direction $\mathbf{d}$ even though the gradients in (9)-(11) change rapidly due to the above-mentioned interactions.

Since any choice of $\phi_{k}$ will satisfy (15), a straightforward choice of $\mathbf{B}^{(k+1)}$ can be obtained from the following additional subproblem which must be solved in each iteration

$$
\begin{array}{ll}
\min _{\phi_{k}} & \operatorname{cond}\left(\mathbf{B}^{(k+1)}\right)=f\left(\mathbf{B}^{(k)}, \mathbf{p}^{(k)}, \mathbf{q}^{(k)}, \phi_{k}\right) \\
\text { s.t. } & \lambda_{1}\left(\mathbf{B}^{(k+1)}\right)=\lambda_{\min }>0
\end{array}
$$

Another update scheme can be obtained if the parameter $\phi_{k}$ in the Broyden formula is kept fixed, while the scalar $\theta_{k}$ in (17) is variable. In the limit $\theta_{k}=0$, it is seen from (17)-(18) that the BFGS formula results in the trivial result $\mathbf{B}^{(k+1)}=\mathbf{B}^{(k)}$. Thus, the purpose of the 
following additional subproblem is to reduce the influence of the relaxation term $\mathbf{B}^{(k)} \mathbf{p}^{(k)}$ in (17) subject to the constraints that $\mathbf{B}^{(k+1)}$ is positive definite and the increase in the condition number is less than a specified factor, say const $=\max (n, 10)$, i.e.

$$
\begin{array}{cl}
\min _{\theta_{k}} & \left(1-\theta_{k}\right) \\
\text { s.t. } & \lambda_{1}\left(\mathbf{B}^{(k+1)}\right)=\lambda_{\min }>0 \\
& \text { const }-\operatorname{cond}\left(\mathbf{B}^{(k+1)}\right) / \operatorname{cond}\left(\mathbf{B}^{(k)}\right)>0 \\
& 0 \geq \theta_{k} \geq 1
\end{array}
$$

Considering highly non-linear problems, information from the very first iterations may differ significantly from the last iterations - especially after major interactions. Pursuing the idea of discarding obsolete information, $\mathbf{B}^{(k+1)}$ can be constructed from information from the last $K$ iterations only. Utilizing this approach, the Hessian matrix is first initialized to I after which the quasi-Newton update formula is applied successively $K$ times - see also Pedersen \& Thoft-Christensen (1993).

At the present stage, the above-mentioned updating schemes have been tested in various combinations on a few examples only. Compared with the effective algorithm NLPQL, 2-5 extra iterations are generally required in non-interactive tests although quicker convergence has been obtained for some types of problems. Based on these preliminary results, the Hessian matrix update of the form (22)-(25) is chosen for the example in section 6 .

\section{IROS - INTERACTIVE RELIABILITY-BASED OPTIMIZATION SYSTEM}

The interactive optimization is carried out through the a shell called IROS - Interactive Reliability-Based $\underline{\text { Optimization }}$ System. Based on the desirable capabilities of an interactive optimization system outlined in Arora \& Tseng (1988), Arora (1989) and others, IROS is being developed for the purpose of testing various interactive facilities and algorithms. IROS is developed for $\mathrm{PC} / \mathrm{DOS}$ and implemented in the programming language $\mathrm{C} / \mathrm{C}++$.

In brief, the core constituents in IROS can be listed as follows:

- Graphics module, i.e. a superior controlling module in which the user is given the opportunity to define various graphical displays, e.g. the iteration history of the object function, selected constraints, sensitivities, design variables, Lagrange multipliers, convergence parameters, stchastic variables, etc.

- Modification module in which the current value of design variables can be changed, design variables be fixed to given values, simple bounds altered, constraints included/excluded from the active set, convergence parameters or even the optimization algorithm itself can be refined or modified.

- Communication or control module in which the information to and from the exterior optimization module is controlled. After a full iteration graphical displays are updated and, in the case where no interaction is detected or no convergence achieved, the iterative process is continued automatically.

- Exterior optimization module in which the optimization algorithm is implemented, i.e. any suitable optimization algorithm can be used if a proper interface is provided. 


\section{EXAMPLE - PLANE PORTAL FRAME}

In order to illustrate some of the interactive capabilities described in section 5 , this section shows a simple reliability-based optimization of a simple plane frame with I-shaped crosssections. The overall geometry, applied load and cross-section are shown in figure 1 while the design variables $\mathbf{z}$ and stochastic variables $\mathbf{x}$ are listed in table 1 .

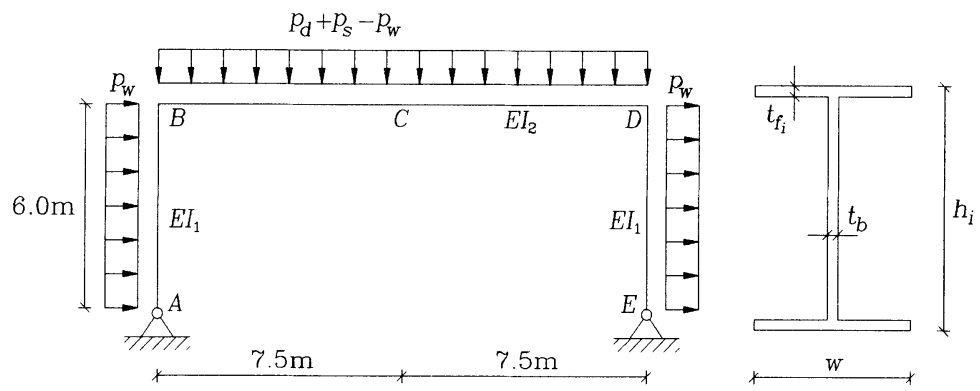

Figure 1: Geometry of frame, applied load and cross-section.

\begin{tabular}{|l|l|c|cc|c|}
\hline \multicolumn{5}{|l|}{ Design Variables } \\
\hline Variable & Designation & Lower & Initial value & Upper \\
\hline$z_{1}=h_{1}$ & Height of cross-section 1 & 200.0 & 250.0 & {$[\mathrm{~mm}]$} & 400.0 \\
$z_{2}=h_{2}$ & Height of cross-section 2 & 200.0 & 300.0 & {$[\mathrm{~mm}]$} & 400.0 \\
$z_{3}=w$ & Width of flange & 75.0 & 100.0 & {$[\mathrm{~mm}]$} & 150.0 \\
$z_{4}=t_{f 1}$ & Thickness of flange 1 & 4.0 & 8.0 & {$[\mathrm{~mm}]$} & 20.0 \\
$z_{5}=t_{f 2}$ & Thickness of flange 2 & 4.0 & 8.0 & {$[\mathrm{~mm}]$} & 20.0 \\
$z_{6}=t_{b}$ & Thickness of body & 4.0 & 6.0 & {$[\mathrm{~mm}]$} & 15.0 \\
\hline Stochastic & Variables & \multicolumn{5}{|c|}{} \\
\hline Variable & Designation & Distrib. & Exp. value & Var. coeff. \\
\hline$x_{1}=f_{y}$ & Yielding strength & LN & $450.0\left[\mathrm{~N} / \mathrm{mm}^{2}\right]$ & 0.08 \\
$x_{2}=E$ & Modulus of elasticity & LN & $206 \cdot 10^{3}$ & {$\left[\mathrm{~N} / \mathrm{mm}^{2}\right]$} & 0.04 \\
$x_{3}=p_{d}$ & Dead load & $\mathrm{N}$ & 3.0 & {$[\mathrm{kN} / \mathrm{m}]$} & 0.05 \\
$x_{4}=p_{s}$ & Applied load, snow & $\mathrm{N}$ & 4.8 & {$[\mathrm{kN} / \mathrm{m}]$} & 0.20 \\
$x_{5}=p_{w}$ & Applied load, wind & $\mathrm{N}$ & 3.0 & {$[\mathrm{kN} / \mathrm{m}]$} & 0.40 \\
\hline
\end{tabular}

Table 1: Design variables $\mathbf{z}$ and stochastic variables $\mathbf{x}$.

In the optimization problem of the standardized form (1)-(4), the volume of the frame is used as the object function $W(\mathbf{z})$ while the reliability-based constraints are related to the bending capacity at 3 critical points, i.e.

$\hat{c}_{j}(\mathbf{z})=\beta_{j}(\mathbf{z})-\beta_{j}^{\min } \geq 0 \quad, \quad j=1,3$

The corresponding $j$ th failure functions can be written as $g_{j}(\mathbf{x})=M_{j}^{u}(\mathbf{x})-M_{j}(\mathbf{x})$, where $M_{j}^{u}(\mathbf{x})$ and $M_{j}(\mathbf{x})$ denote the bending capacity and the moment due to the total applied load, respectively. Due to the direction of the wind, the inequality $\left|M_{B}\right| \geq\left|M_{D}\right|$ always 
holds. Therefore, the 3 failure functions consist of 2 failure functions at point B (column and girder, respectively) and 1 failure function at point $\mathrm{C}$ at the centre of the girder.

In addition, 4 simple deterministic, geometric constraints $\hat{c}_{4}(\mathbf{z})-\hat{c}_{7}(\mathbf{z})$ with respect to local instability are included: 2 constraints requiring that the ratio between the height of the cross-section $h_{j}$ and body thickness $t_{b}$ does not exceed 50 and 2 constraints requiring that the ratio between the width $w$ and the flange thickness $t_{f j}$ does not exceed 20 .

In order to demonstrate some of the interactive capabilities of IROS, the above defined optimization problem is solved according to the following scheme:

1. Optimization where all 6 design variables are allowed to vary and all 7 constraints (3 reliability-based and 4 geometric constraints) are included.

2. Based on the above continuous solution, the 3 design variables that describe thicknesses $\left(t_{f 1}, t_{f 2}\right.$ and $\left.t_{b}\right)$ are fixed to adjacent discrete values. From this point the optimization is continued with the remaining 3 free design variables.

3. Finally, the 4 geometric constraints $\hat{c}_{4}(\mathbf{z})-\hat{c}_{7}(\mathbf{z})$ are excluded and all 6 design variables allowed to vary. A continuous solution prone to local instability is hereby obtained.

The iteration history of design variables, object function and reliability-based constraints are shown at the screen-dump from IROS in figure 2 . In addition, the values of the design variables and object function at the start and the end for the 3 optimizations are listed in table 2 .

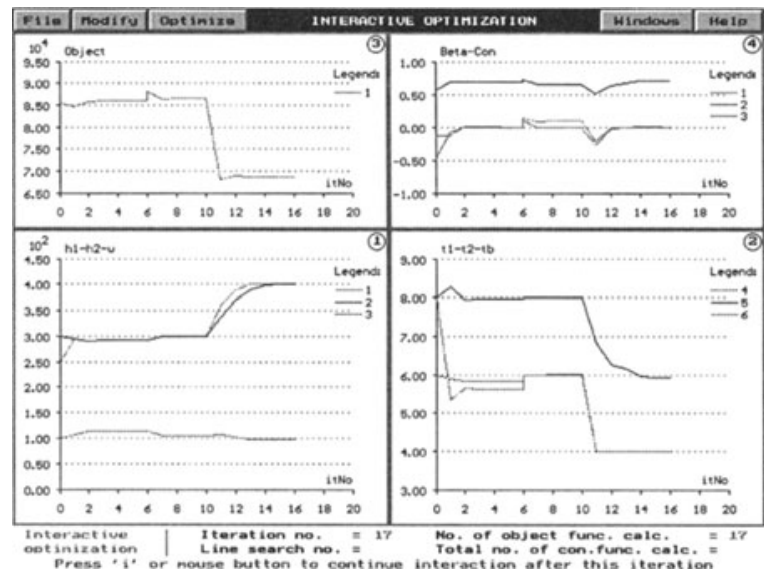

Figure 2: Iteration history of design variables, object function and rel.-based constraints.

From figure 2 and table 2 it is seen, that the continuous solution from the 1st run is obtained after 6 iterations. At this point, the 3 design variables describing thicknesses $\left\{t_{f 1}\right.$, $\left.t_{f 2}, t_{b}\right\}=\{5.63,7.97,5.84\}$ are changed and fixed interactively to the adjacent standard dimensions $\{6.00,8.00,6.00\}$.

After this interactive change the optimization is continued to iteration 10 (2nd run) as shown in figure 2. At this point, a new "discrete" optimum is achieved. A feasible solution with standard dimensions suitable for practical used is hereby obtained with an increase in the object function by less than $1 \%$. 


\begin{tabular}{|l|l|c|c|c|c|c|c|}
\hline \multicolumn{2}{|l|}{ Optimization (iteration no.) } & \multicolumn{2}{|c|}{ 1st run (1-6) } & \multicolumn{2}{c|}{ 2nd run (7-10) } & \multicolumn{2}{c|}{ 3rd run (11-17) } \\
\hline Variable & Designation & Start & End & Start & End & Start & End \\
\hline$z_{1}=h_{1}$ & Height of cross-sec. 1 & 250.0 & 291.8 & 291.8 & 300.0 & 300.0 & 400.0 \\
$z_{2}=h_{1}$ & Height of cross-sec. 2 & 300.0 & 291.8 & 291.8 & 300.0 & 300.0 & 400.0 \\
$z_{3}=w$ & Width of flange & 100.0 & 112.6 & 112.6 & 104.6 & 104.6 & 96.7 \\
$z_{4}=t_{f 1}$ & Thickness of flange 1 & 8.00 & 5.63 & 6.00 & 6.00 & 6.00 & 4.00 \\
$z_{5}=t_{f 2}$ & Thickness of flange 2 & 8.00 & 7.97 & 8.00 & 8.00 & 8.00 & 5.91 \\
$z_{6}=t_{b}$ & Thickness of body & 6.00 & 5.84 & 6.00 & 6.00 & 6.00 & 4.00 \\
\hline \multicolumn{2}{|l|}{ Object function } & - & 85.9 & - & 86.5 & - & 68.5 \\
\hline
\end{tabular}

Table 2: Design variables $\mathbf{z}$ during the 3 stages of the optimization.

Finally, all design variables are relaxed and allowed to vary in the 3rd optimization, where only the 3 reliability-based constraints are included. Thus, based on the information obtained in the first 10 iterations, the optimization is now continued for another 7 iterations as shown in figure 2. A dramatically change in the design and decrease of the object function by approximately $20 \%$ is hereby obtained even though 4 of the 6 design variables have reached the simple bounds.

\section{ACKNOWLEDGEMENT}

This paper is supported by the research project "Marine Structural Design" sponsored by the Danish Technical Research Council.

\section{REFERENCES}

[1] Arora, J.S.: Introduction to Optimum Design, McGraw-Hill, 1989.

[2] Arora, J.S. \& C.H. Tseng: "Interactive Design Optimization", Engineering Optimization, Vol. 13, 1988, pp. 173-188.

[3] Gill, P.E., W. Murray \& M.H. Wright: Practical Optimization, Academic Press, 1981.

[4] Golub, G.H. \& C.F. Van Loan: Matrix Computations, 2nd edition, Johns Hopkins University Press, 1989.

[5] Luenberger, D.G.: Linear and Nonlinear Programming, 2nd edition, Addison-Wesley, Reading, Massachusetts, 1984.

[6] Madsen, H.O., S. Krenk \& N.C. Lind (1986): Methods of Structural Safety, PrenticeHall, Englewood Cliffs, New Jersey, 1986.

[7] Pedersen, C. \& P. Thoft-Christensen: "Interactive Quasi-Newton Optimization Algorithms", Structural Reliability Theory, Paper No. 120, ISSN 0902-7513 R9346, University of Aalborg, Denmark, 1993.

[8] Powell, M.J.D.: "A Fast Algorithm for Nonlinear Constrained Optimization Calculations", Lecture Notes in Mathematics, No. 630, Numerical Analysis, Proceedings, Biennial Conference, Dundee 1977, pp. 144-157, Springer-Verlag, 1977.

[9] Ringertz, U.T.: Numerical Optimization: With Applications in Structural Analysis and Design, Lecture Notes, Vol. 1, Comett Course: Computer Aided Optimum Design of Strucures, Institute of Mechanical Engineering, Aalborg University, Aug. 1993. 